\title{
Slow Frequency-Hopping Multicarrier DS-CDMA for Transmission over Nakagami Multipath Fading Channels
}

\author{
Lie-Liang Yang, Member, IEEE, and Lajos Hanzo, Senior Member, IEEE
}

\begin{abstract}
A novel multiple access scheme based on slow frequency hopping multicarrier direct-sequence code division multiple access (SFH/MC DS-CDMA) is proposed and investigated, which can be rendered compatible with the existing second-generation narrowband CDMA and third-generation wideband CDMA systems. The frequency hopping patterns are controlled by a set of constant-weight codes. Consequently, multirate communications can be implemented by selecting the corresponding sets of constant-weight codes having the required weights controlling the SFH patterns invoked. Two FH schemes, namely random and uniform $\mathrm{FH}$, are considered and their advantages as well as disadvantages are investigated. We assume that the system operates in a multipath fading environment and a RAKE receiver structure with maximum ratio combining (MRC) is used for demodulation. The system's performance is evaluated over the range of multipath Nakagami fading channels, under the assumption that the receiver has an explicit knowledge of the associated frequency-hopping $(\mathrm{FH})$ patterns invoked. Furthermore, the performance of the SFH/MC DS-CDMA system is compared to that of the conventional single-carrier (SC) DS-CDMA system and that of the conventional MC DS-CDMA system, under the assumptions of constant system bandwidth and of constant transmitted signal power.
\end{abstract}

Index Terms-Code division multiple access, constant-weight code, frequency hopping, Nakagami fading, orthogonal frequency division multiplexing.

\section{INTRODUCTION}

W ITH THE EMERGENCE of broadband radio access networks (BRAN) requiring bandwidths substantially higher than that of the third generation $(3 \mathrm{G})$ systems [1], it is of increasing significance to create a BRAN framework, which is backward compatible with second generation $(2 \mathrm{G})$ and $3 \mathrm{G}$ schemes. An attractive candidate scheme capable of achieving these requirements is constituted by the proposed frequency-hopping (FH) based multicarrier DS-CDMA (FH/MC DS-CDMA) [2], [3] arrangement, where no rigid spectral segmentation is required for the coexistence of various wireless systems. Specifically, the entire bandwidth can be divided into a number of subbands and each subband can be assigned a subcarrier [4]-[8]. A subcarrier could deliver a narrowband IS-95 type service or-similarly to the emerging

Manuscript received April 29, 1999; revised June 12, 2000. This work was supported by the EPSRC, U.K., and by the Commission of the European Communities, Brussels, Belgium.

The authors are with the Department of Electronics and Computer Science, University of Southampton, SO17 1BJ Southampton, U.K.

Publisher Item Identifier S 0733-8716(01)04704-7. multicarrier-assisted CDMA2000 system [9]-it could invoke a number of carriers, while employing a variety of different spreading factors [1], for example.

FH can be invoked for each user, in order to occupy the whole system bandwidth and to utilize efficiently the system's frequency resources. Various slow FH, fast FH, or adaptive FH techniques can be supported, depending on the state-of-the-art. In FH/MC DS-CDMA systems, the subbands are not required to be of equal bandwidth. Hence, existing $2 \mathrm{G}$ and $3 \mathrm{G}$ CDMA systems can be supported using one or more subcarriers, consequently simplifying the frequency resource management and efficiently utilizing the entire bandwidth available. Furthermore, the FH/MC DS-CDMA system concept lends itself to unlicensed operation, eradicating the inefficient spectral segmentation of different coexisting systems.

The performance of FH/MC DS-CDMA systems using blind joint soft-detection has been investigated in [2] and [3], under assumption that the receiver has no knowledge of the associated FH patterns invoked. It was shown that blind joint soft-detection can achieve the required bit-error-rate (BER) performance, while blindly acquiring the FH patterns employed. In this treatise, we study a FH/MC DS-CDMA system employing slow FH [10], [11]—resulting in a SFH/MC DS-CDMA system—and binary modulation. The system operates in a multipath fading environment, and a maximum ratio combining (MRC) assisted RAKE receiver is used. The system's performance is evaluated over the range of Nakagami- $m$ multipath fading channels, which closely model various multipath channels, exhibiting probability density functions (pdf) spanning the range from Rayleigh fading channels to nonfading Gaussian channels by varying a single parameter, namely $m$, from one to infinity [12]-[20]. In contrast to [3], in this paper, the performance of the proposed SFH/MC DS-CDMA system is investigated and compared to that of conventional single-carrier DS-CDMA (SC DS-CDMA) systems as well as to conventional MC DS-CDMA systems actively using all the available subcarriers, under the assumption that the receiver of the SFH/MC DS-CDMA scheme has the explicit knowledge of the FH patterns. Specially, two FH schemes are considered. The first is a random FH scheme, where each user hops independently according to its FH patterns. By contrast, in the second scheme, each user's FH pattern relies on explicit side information, in order that all subcarriers of the system are uniformly occupied and that the multiuser interference is minimized. Hence, in our forthcoming discourse the former $\mathrm{FH}$ scheme is referred to random $\mathrm{FH}$, while the latter as uniform FH. 


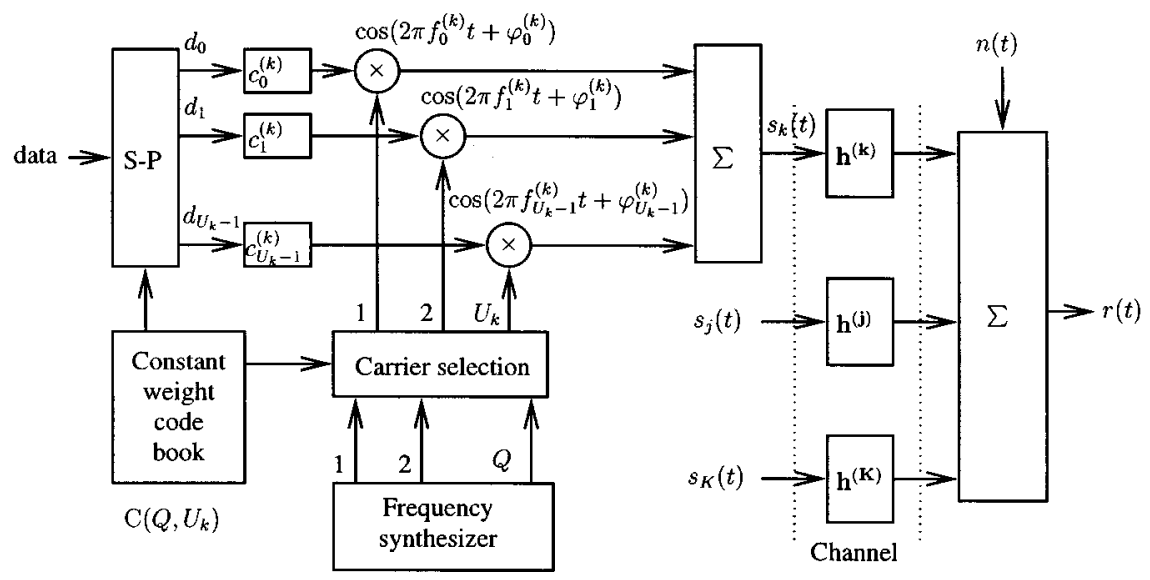

Fig. 1. Transmitter and channel block diagram of the FH/MC DS-CDMA system.

The remainder of this paper is organized as follows. In Section II, we discuss the proposed SFH/MC DS-CDMA system, which includes the description of the transmitted signal, the channel model and the receiver model. In Section III, we derive BER expressions for the proposed system associated with both random and uniform FH. Our numerical results and discussions are provided in Section IV, and finally, our conclusion is offered in Section V. Let us now consider the proposed system.

\section{SFH/MC DS-CDMA SYSTEM}

\section{A. Transmitter Model}

The transmitter schematic along with the multiple access channel is shown in Fig. 1. There are $K$ users in the system and each user is assigned a random spreading sequence. In the schematic, $\left(Q, U_{k}\right)$ denotes a constant-weight code assigned to user $k$, which has $U_{k}$ number of binary '1's, indicating the activated FH carriers. Hence, the weight of $\left(Q, U_{k}\right)$ is $U_{k}$. This code is selected from the constant-weight code book of the schematic, which describes the FH patterns. There are $\left(\begin{array}{c}Q \\ U_{k}\end{array}\right)=Q ! / U_{k} !\left(Q-U_{k}\right) !$ number of possible such weight- $U_{k}$ sequences and the constant-weight code $\mathrm{C}\left(Q, U_{k}\right)$ describes the FH patterns employed. Specifically, $\left(Q, U_{k}\right)$ determines the positions of the $U_{k}$ number of binary ' 1 's, which control the selection of a set of $U_{k}$ number of active $\mathrm{FH}$ subcarrier frequencies from the $Q$ outputs of the frequency synthesizer in Fig. 1.

The original bit stream having a bit duration of $T_{b}$ is first serial-to-parallel (S-P) converted, as seen in the schematic of Fig. 1 , yielding $U_{k}$ parallel streams. Let us denote the bit duration of each parallel stream by $T$, which represents the symbol duration of the SFH/MC DS-CDMA signals. Then, if the system is designed in order to mitigate the intersymbol-interference (ISI) in a constant-rate transmission scheme, the symbol duration is extended and becomes $T=U_{k} T_{b}$. By contrast, if the design aims to support multiple information rates, a constant bit duration of $T=T_{b}$ can be employed, and multirate transmissions are implemented by employing a different number of subcarriers, as we will highlight during our further discourse. Theoretically, $Q$ number of different information rates can be achieved by changing the weight, $U_{k}$, of the code $\mathrm{C}\left(Q, U_{k}\right)$ from 1 to $Q$.
As seen in Fig. 1, the direct-sequence (DS) spread, transmitted signal of the $k$ th user can be formulated as

$$
\begin{aligned}
s_{k}(t)= & \sum_{u_{k}=0}^{U_{k}-1} \sqrt{2 P} d_{u_{k}}^{(k)}(t) c_{u_{k}}^{(k)}(t) \\
& \cdot \cos \left(2 \pi f_{u_{k}}^{(k)} t+\varphi_{u_{k}}^{(k)}\right),
\end{aligned}
$$

where $P$ denotes the transmitted power per carrier, while $U_{k}$ is the weight of the constant-weight FH code of the $k$ th user. Furthermore, $\left\{d_{u_{k}}^{(k)}(t)\right\},\left\{c_{u_{k}}^{(k)}(t)\right\},\left\{f_{u_{k}}^{(k)}\right\}$ and $\left\{\varphi_{u_{k}}^{(k)}\right\}$ denote the current data stream's waveforms, the DS spreading waveforms, the subcarrier frequency set and the phase angles introduced in the carrier modulation process. Let $T_{c}$ be the chip duration of the DS spreading waveforms and $N=T_{b} / T_{c}$. Then, the processing gain of $N_{P}=T / T_{c}$ equals to $U_{k} N$ or $N$, depending on the choice of the symbol duration, as discussed previously. Furthermore, we assume that the FH interval is $T_{h}$, and that the number of data bits, $N_{b}=T_{h} / T$, transmitted per hop is a positive integer, which is strictly larger than 1, implying slow FH.

\section{B. Nakagami Channel Model}

We considered a frequency-selective multipath fading channel [21], whose complex low-pass impulse response for subcarrier $u_{k}$ of user $k$ is given by

$$
h_{u_{k}}^{(k)}(t)=\sum_{l_{p}=0}^{L_{p}-1} \alpha_{u_{k}, l_{p}}^{(k)} e^{j \phi_{u_{k}, l_{p}}^{(k)}} \delta\left(t-l_{p} T_{c}\right),
$$

where $l_{p} T_{c}$ is the relative delay of the $l_{p}$ th path of the $u_{k}$ th subcarrier of user $k$ with respect to the main path, the phases $\left\{\phi_{u_{k}, l_{p}}^{(k)}\right\}$ are independent identically distributed (i.i.d.) random variables uniformly distributed in the interval $[0,2 \pi)$, whilst the $L_{p}$ tap weights $\left\{\alpha_{u_{k}, l_{p}}^{(k)}\right\}$ are independent Nakagami- $m$ random variables with a pdf of [15]:

$$
\begin{aligned}
p\left(\alpha_{u_{k}, l_{p}}^{(k)}\right) & =M\left(\alpha_{u_{k}, l_{p}}^{(k)}, m, \Omega_{u_{k}, l_{p}}^{(k)}\right), \\
M(R, m, \Omega) & =\frac{2 R^{2 m-1}}{\Gamma(m)}\left(\frac{m}{\Omega}\right)^{m} e^{(-m / \Omega) R^{2}}
\end{aligned}
$$

where $\Gamma(\cdot)$ represents the gamma function, and $m$ is the Nakagami- $m$ fading parameter, which is defined as 


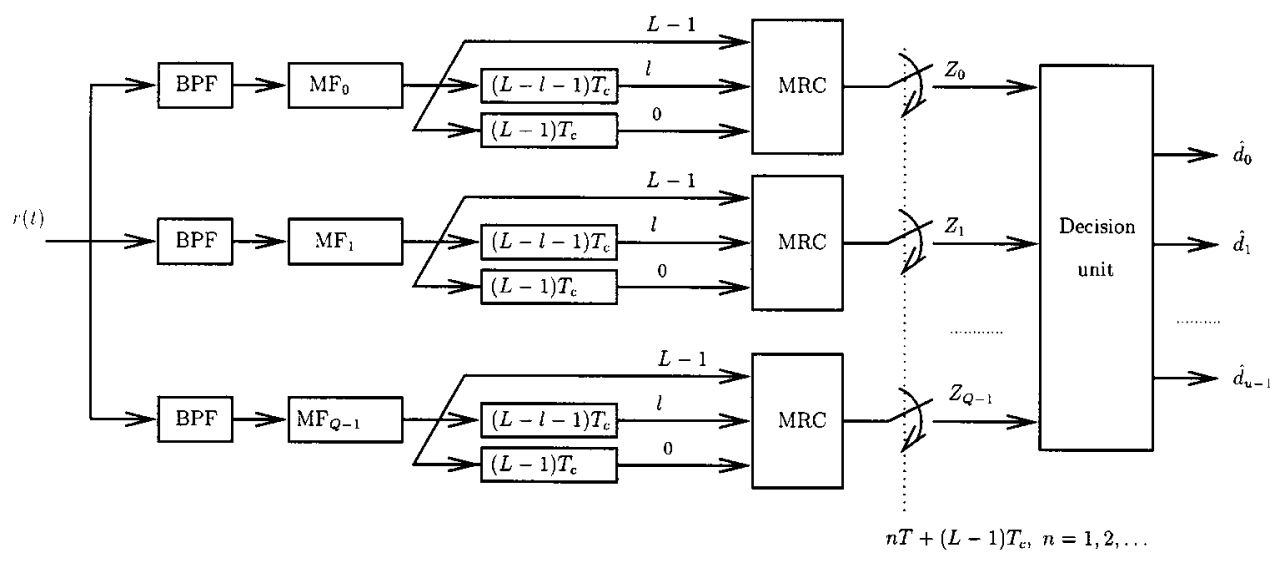

Fig. 2. Receiver block diagram of the FH/MC DS-CDMA system.

$m=E^{2}\left[\left(\alpha_{u_{k}, l_{p}}^{(k)}\right)^{2}\right] / \operatorname{Var}\left[\left(\alpha_{u_{k}, l_{p}}^{(k)}\right)^{2}\right]$. The parameter $m$ of the amplitude distribution characterizes the severity of the fading on the $l_{p}$ th resolvable path [12]. For instance, $m=1 / 2$ and $m=1$ correspond to the one-sided Gaussian fading and the well-known Rayleigh fading. In the limit, as $m \rightarrow \infty$, the Nakagami- $m$ fading channel converges to a nonfading additive white gaussian noise (AWGN) channel. Furthermore, the Ricean and lognormal distributions can also be closely approximated by the Nakagami distribution for $m>1$. In general, the following relationship exists between the Ricean factor $\mathcal{K}$ and the parameter $m$ [12]

$$
m \approx \frac{1}{1-\left(\frac{\mathcal{K}}{1+\mathcal{K}}\right)^{2}}
$$

where the Ricean factor $\mathcal{K}$ is defined as the ratio of the average direct power and the average scattered power. The parameter $\Omega_{u_{k}, l_{p}}^{(k)}$ in (3) is the second moment of $\alpha_{u_{k}, l_{p}}^{(k)}$, i.e., $\Omega_{u_{k}, l_{p}}^{(k)}=E\left[\left(\alpha_{u_{k}, l_{p}}^{(k)}\right)^{2}\right]$. We assume a negative exponentially decaying multipath intensity profile (MIP) distribution given by

$$
\Omega_{u_{k}, l_{p}}^{(k)}=\Omega_{u_{k}, 0}^{(k)} e^{-\eta l_{p}}, \quad \eta \geq 0
$$

where $\Omega_{u_{k}, 0}^{(k)}$ is the average signal strength corresponding to the first resolvable path and $\eta$ is the rate of average power decay. Consequently, for an asynchronous CDMA system with $K$ users, the received signal takes the form:

$$
\begin{aligned}
r(t)= & n(t)+\sqrt{2 P} \sum_{k=1}^{K} \sum_{u_{k}=0}^{U_{k}-1} \sum_{l_{p}=0}^{L_{p}-1} \alpha_{u_{k}, l_{p}}^{(k)} d_{u_{k}}^{(k)}\left(t-l_{p} T_{c}-\tau_{k}\right) \\
& \cdot c_{u_{k}}^{(k)}\left(t-l_{p} T_{c}-\tau_{k}\right) \cos \left(2 \pi f_{u_{k}}^{(k)} t+\psi_{u_{k}, l_{p}}^{(k)}\right)
\end{aligned}
$$

where $n(t)$ represents the AWGN with zero mean and doublesided power spectral density of $N_{0} / 2$,

$$
\psi_{u_{k}, l_{p}}^{(k)}=\left[\varphi_{u_{k}}^{(k)}+\phi_{u_{k}, l_{p}}^{(k)}-2 \pi f_{u_{k}}^{(k)}\left(\tau_{k}+l_{p} T_{c}\right)\right](\bmod 2 \pi)
$$

which is assumed to be an i.i.d. random variable having a uniform distribution in $[0,2 \pi)$, and $\tau_{k}$ represents the propagation delay of user $k$.

\section{Receiver Model}

Let the first user be the user-of-interest and consider the conventional matched filter based RAKE receiver with maximal ratio combining (MRC), as shown in Fig. 2, where the superscript and subscript of the reference user's signal has been omitted for notational convenience. In Fig. $2 L$ number of diversity branches are used by the receiver, where $1 \leq L \leq L_{p}$ is a variable, allowing us to study the effect of different diversity orders. Upon exploiting the explicit knowledge of the FH patterns, detection can be implemented by demodulating only the $U_{k}$ active subcarriers. Alternatively, all $Q$ subcarriers can always be demodulated, while only $U_{k}$ MRC outputs selected according to the FH patterns are used for detection. Furthermore, in order to invoke the fast Fourier transform (FFT) for modulation/demodulation, each subcarrier can be viewed as an ON-OFF type signaling scheme, and all the $Q$ number of subcarriers can be processed. When a subcarrier is actively used for signaling and, hence, it is in the ON-state, the MRC output samples give +1 or -1 information, while when passive and hence in the OFF-state, the MRC stage outputs noise. Finally, the active MRC outputs can be demodulated by exploiting the knowledge of the FH patterns involved, which can be achieved without invoking frequency dehopping at the receiver. Therefore, the receiver of the proposed SFH/MC DS-CDMA system has a similar complexity to that of the corresponding conventional MC DS-CDMA system using all subcarriers for transmission.

The $Q$ number of matched filters in Fig. 2 are matched to the reference user's spreading codes in the subbands associated with the $Q$ number of subcarriers, and are assumed to be perfectly time-synchronized with the corresponding initial paths of the $Q$ number of subcarriers of the reference signal. If we assume that perfect estimates of the channel tap weights and phases are available, then after appropriately delaying the individual matched filter outputs, in order to synchronize the $L$ number of path signals used by the RAKE combiner, the $q$ th MRC output sampled at $t=n T+(L-1) T_{c}$, in order to detect the $n$th symbol can be expressed as

$$
Z_{q}[n]=\sqrt{\frac{P}{2}} T\left(D_{q}[n]+I_{q}\right)
$$


where $D_{q}[n]$ represents the desired component, which can be expressed as

$$
D_{q}[n]=d_{q}(n) \sum_{l=0}^{L-1} \alpha_{q, l}^{2}
$$

and $d_{q}(n)$ is the $n$th bit transmitted on subcarrier $q$ by the reference user, while $d_{q}(n) \in\{+1,-1,0\}$ with " 0 " representing the OFF-state. Since we have assumed that there exists no interference among the subcarriers-i.e., interference is inflicted only, when an interfering user activates the same subcarrier, as the reference user-the interference plus noise term $I_{q}$ in (7) can be expressed as

$$
I_{q}=I_{q}[S]+I_{q}[M]+N_{q},
$$

where $I_{q}[S]$ is contributed by the multipath interference due to the $q$ th subcarrier of the user-of-interest, which can be expressed as

$$
\begin{aligned}
I_{q}[S]= & \sum_{l=0}^{L-1} \alpha_{q, l} \sum_{\substack{l_{p}=0 \\
l_{p} \neq l}}^{L_{p}-1} \frac{\alpha_{q, l_{p}} \cos \theta_{q, l_{p}}}{T} \int_{(L-1) T_{c}}^{T+(L-1) T_{c}} \\
& \cdot d_{q}\left[t-\left(L+l_{p}-l-1\right) T_{c}\right] \\
& \cdot c_{q}\left[t-\left(L+l_{p}-l-1\right) T_{c}\right] c_{q}\left[t-(L-1) T_{c}\right] d t
\end{aligned}
$$

where $\theta_{q, l_{p}}=\psi_{q, l_{p}}-\psi_{q, l} . I_{q}[M]$ in (9) is contributed by the multiple access interference (MAI) inflicted by the $q$ th subcarrier of the interfering users. Let us assume that there are $K_{h}$ $\left(0 \leq K_{h} \leq K-1\right)$ number of interfering signals, all of which activate the $q$ th subcarrier during the $n$th symbol of the reference signal. The event, when an interferer uses the same subcarrier as the reference user, is often referred to in the literature as a so-called hit [10] — an issue that will be discussed in detail in the next section. Then, $I_{q}[M]$ can be expressed as

$$
\begin{aligned}
I_{q}[M]= & \sum_{l=0}^{L-1} \alpha_{q, l} \sum_{h=1}^{K_{h}} \sum_{l_{p}=0}^{L_{p}-1} \frac{\alpha_{q, l_{p}}^{(h)} \cos \theta_{q, l_{p}}^{(h)}}{T} \int_{(L-1) T_{c}}^{T+(L-1) T_{c}} \\
& \cdot d_{q}^{(h)}\left[t-\left(L+l_{p}-l-1\right) T_{c}-\tau_{h}\right] \\
& \cdot c_{q}^{(h)}\left[t-\left(L+l_{p}-l-1\right) T_{c}-\tau_{h}\right] \\
& \cdot c_{q}\left[t-(L-1) T_{c}\right] d t .
\end{aligned}
$$

where $\theta_{q, l_{p}}^{(h)}=\psi_{q, l_{p}}^{(h)}-\psi_{q, l}$. Finally, the noise term of (9) can be expressed as

$$
\begin{aligned}
N_{q}= & \left(\sqrt{\frac{P}{2}} T\right)^{-1} \sum_{l=0}^{L-1} \alpha_{q, l} \int_{(L-1) T_{c}}^{T+(L-1) T_{c}} \\
& \cdot n(t) c_{q}\left[t-(L-1) T_{c}\right] \cos \left(2 \pi f_{q} t+\psi_{q, l}\right) d t
\end{aligned}
$$

which is a Gaussian random variable with zero mean and variance of $\left(N_{0} / 2 E_{b}\right) \sum_{l=0}^{L-1} \alpha_{q, l}^{2}$, where $\left\{\alpha_{q, l}\right\}$ represents the path attenuations and $E_{b}=P T$ represents the average transmitted energy-per-bit.

We assume that the MAI and the self-interference terms of (9) can be modeled as an additive Gaussian noise process with zero mean and a variance equal to the sum of the corresponding variances. Note that the Gaussian approximation typically becomes sufficiently accurate in the context of CDMA, when the number of users is in excess of $K=8$, provided that the BER is higher than $10^{-6}$ [23]. Hence, for a set of given channel amplitudes $\left\{\alpha_{q, l}\right\}$, the $q$ th MRC output sample can be approximated as a Gaussian variable having a normalized mean value given by (8) and a normalized variance given by [15], [17]

$$
\begin{aligned}
\sigma^{2}= & {\left[\frac{1}{2 N_{p}} \sum_{\substack{l_{p}=0 \\
l_{p} \neq l}}^{L_{p}-1} \Omega_{q, l_{p}}+\frac{1}{3 N_{p}} \sum_{h=1}^{K_{h}} \sum_{l_{p}=0}^{L_{p}-1} \Omega_{q, l_{p}}^{(h)}+\left(\frac{2 E_{b}}{N_{0}}\right)^{-1}\right] } \\
& \cdot \sum_{l=0}^{L-1} \alpha_{q, l}^{2}
\end{aligned}
$$

where the first square-bracketed term is contributed by the selfinterference given by (10), the second term is by the MAI expressed in (11), while the third term is by the noise of (12). In order to reduce the number of parameters involved, the usual assumption is that $\Omega_{q, l_{p}}^{(h)}=\Omega_{0} e^{-\eta l_{p}}$, which means that all the users have the same average signal power at the receiver as a result of perfect power-control. Then, (13) can be simplified to

$$
\begin{gathered}
\sigma^{2} \approx\left[\frac{q\left(L_{p}, \eta\right)}{2 N_{p}}\left(\frac{L_{p}-1}{L_{p}}\right)+\frac{K_{h} q\left(L_{p}, \eta\right)}{3 N_{p}}\right. \\
\left.+\left(\frac{2 \Omega_{0} E_{b}}{N_{0}}\right)^{-1}\right] \Omega_{0} \sum_{l=0}^{L-1} \alpha_{q, l}^{2}
\end{gathered}
$$

where

$$
\begin{aligned}
q\left(L_{p}, \eta\right) & =\sum_{l_{p}=0}^{L_{p}-1} e^{-\eta l_{p}} \\
& =\left(1-e^{-\eta L_{p}}\right) /\left(1-e^{-\eta}\right), \quad \text { if } \eta \neq 0
\end{aligned}
$$

otherwise, $q\left(L_{p}, \eta\right)=L_{p}$, if $\eta=0$. Note that in order to simplify (13) to (14), we approximated the $\Omega_{q, l}$ term by its average value of $q\left(L_{p}, \eta\right) \Omega_{0} / L_{p}$.

\section{Average Bit ERror Probability}

In the last section, we have analyzed the statistics of the MRC output samples. Let us now analyze the BER performance of the proposed system in the context of random FH and uniform FH schemes.

\section{A. Random FH Scheme}

In our proposed SFH/MC DS-CDMA system using random $\mathrm{FH}$, the subcarriers associated with each transmission of the $k$ th user, where $k=1,2, \ldots, K$, are determined by the preassigned FH patterns constituting a group of constant-weight codewords. The active subcarriers are switched from a group of frequencies to another without the knowledge of the $\mathrm{FH}$ patterns of the other users. The advantage of the proposed SFH/MC DS-CDMA system using random FH is that-similarly to hybrid DS/FH CDMA systems [10], [11]—it can combine the best features of DS and FH spread spectrum modulation schemes, while 
avoiding many of their individual shortcomings. However, the number of users activating each subcarrier is a random variable ranging from zero to $K$ for the SFH/MC DS-CDMA system using the random $\mathrm{FH}$ scheme. Hence, in this context multiuser detection algorithms associated with a variable number of active users must be investigated, if multiuser detection [22] has to be invoked.

In the performance evaluation of DS/SFH CDMA systems, the average BER can be computed by first evaluating the conditional error probability given the number of "hits," where a "hit" implies that an interfering signal activates the same frequency subcarrier as the user-of-interest. Otherwise, when the interfering users activate different frequency subcarriers, there will be no MAI inflicted upon the activated subcarrier of the reference signal, as we assumed previously. After obtaining the conditional BER, the unconditional BER can be computed by averaging the conditional BER with respect to the distribution of hits. The BER of the proposed SFH/MC DS-CDMA system using random FH can be computed following the above approach.

Since the receiver has the explicit knowledge of the FH pattern of the transmitter, the information transmitted on the $U$ number of activated carriers can be decided without taking the OFF-state carriers into account. Hence, the average BER can be expressed as [10], [11]

$$
P_{b}=\sum_{K_{h}=0}^{K-1}\left(\begin{array}{c}
K-1 \\
K_{h}
\end{array}\right) P_{h}^{K_{h}}\left(1-P_{h}\right)^{K-1-K_{h}} P_{b}\left(K_{h}\right)
$$

where $0 \leq K_{h} \leq K-1$ and $P_{h}$ is the probability of a hit-as defined above-from an interfering signal. In an asynchronous system, if we assume that the FH pattern is determined randomly by a constant-weight code chosen from the set of $\left(\begin{array}{c}Q \\ U_{k}\end{array}\right)$ codes and assume that the number of bits, $N_{b}$, transmitted during each FH interval satisfies $N_{b} \gg 1$, then the probability of a hit from the interfering user $k$ can be approximated by:

$$
P_{h}(k) \approx \frac{\left(\begin{array}{c}
Q-1 \\
U_{k}-1
\end{array}\right)}{\left(\begin{array}{c}
Q \\
U_{k}
\end{array}\right)}=\frac{U_{k}}{Q}
$$

where $U_{k}$ represents the weight of the constant-weight codes employed. Furthermore, if the SFH/MC DS-CDMA system was designed to be capable of supporting multirate services, the average probability of a hit, $P_{h}$, can be computed by averaging (16). Let us assume that the SFH/MC DS-CDMA system can support $M, 1 \leq M \leq Q$, different information rates corresponding to $U_{1}, U_{2}, \ldots, U_{M}$ number of subcarriers. We assume furthermore that each user transmits independently, using any one of the $M$ information rates with the same probability. Then, the average probability of a hit can be expressed as

$$
P_{h}=\frac{1}{Q M} \sum_{i=1}^{M} U_{i}
$$

The probability of $P_{b}\left(K_{h}\right)$ in (15) denotes the conditional bit error probability, given that $K_{h}$ hits occurred from the other $K-1$ interfering users, i.e $K_{h}$ out of $K-1$ users in the system activated the same subcarrier as the reference user.

Before proceeding to the evaluation of the average BER of $P_{b}\left(K_{h}\right)$ for a given $K_{h}$, we first have to find the BER conditioned on the multipath component attenuation $\left\{\alpha_{q, l}\right\}$ for a given $K_{h}$. Following the discussions of the last subsection on the statistics of the decision variables, for the receiver having an explicit knowledge of the FH pattern, the associated conditional BER may be written as

$$
P_{b}\left(K_{h},\left\{S_{l}\right\}_{l=0}^{L-1}\right)=Q\left(\sqrt{\bar{\gamma} \sum_{l=0}^{L-1} S_{l}}\right)
$$

where $Q(\cdot)$ is the $Q$-function defined as [21]

$$
Q(x)=\frac{1}{\sqrt{2 \pi}} \int_{x}^{\infty} e^{-t^{2} / 2} d t .
$$

Alternatively, the Gaussian $Q$-function can be represented as [13]:

$$
Q(x)=\frac{1}{\pi} \int_{0}^{\pi / 2} \exp \left(-\frac{x^{2}}{2 \sin ^{2} \theta}\right) d \theta, \quad x \geq 0 .
$$

The latter $Q$-function representation has the advantage of having finite integration limits that are independent of the argument $x$. Hence (19) allows us to obtain a convenient analytical BER expression for the scenario, where the multipath channel outputs are not identically distributed [13], which is the case considered in this paper, since the exponentially decaying MIP distribution of (5) was assumed. In (18), $\bar{\gamma}$ represents the average received signal-to-noise ratio (SNR) given by

$$
\bar{\gamma}=\left[\frac{q\left(L_{p}, \eta\right)}{2 N_{p}}\left(\frac{L_{p}-1}{L_{p}}\right)+\frac{K_{h} q\left(L_{p}, \eta\right)}{3 N_{p}}+\left(\frac{2 \Omega_{0} E_{b}}{N_{0}}\right)^{-1}\right]^{-1}
$$

while $S_{l}, l=0,1, \ldots, L-1$-the normalized square of the Nakagami distributed multipath attenuations-is expressed as

$$
S_{l}=\frac{\alpha_{q, l}^{2}}{\Omega_{0}}
$$

The average BER for a given $K_{h}$ can be calculated from the conditional error probability upon weighting $P_{b}\left(K_{h},\left\{S_{l}\right\}_{l=0}^{L-1}\right)$ by the joint pdf of $\left\{S_{l}\right\}_{l=0}^{L-1}$, namely by $p_{S_{0}, S_{1}, \ldots, S_{L-1}}\left(s_{0}, s_{1}, \ldots, s_{L-1}\right)$, and then averaging or integrating the weighted product over its legitimate range, as it will be made explicit below. Since $\left\{\alpha_{q, l}\right\}$ is a Nakagami-distributed variable having a pdf of $M\left(\alpha_{q, l}, m, \Omega_{q, l}\right)$, as seen in (3), $S_{l}=\alpha_{q, l}^{2} / \Omega_{0}$ is a Gamma distributed random variable having a pdf given by [20]

$$
p_{S_{l}}(s)=\frac{s_{l}^{m-1}}{\Gamma(m)}\left(\frac{m}{\lambda_{l}}\right)^{m} e^{\left(-m / \lambda_{l}\right) s_{l}}, \quad s_{l} \geq 0
$$


where $\lambda_{l}=\Omega_{l} / \Omega_{0}=e^{-\eta l}, \Gamma(\cdot)$ is the Gamma function defined as $\Gamma(z)=\int_{0}^{\infty} e^{-t} t^{z-1} d t, z>0$. Since the random variables $\left\{S_{l}\right\}_{l=0}^{L-1}$ are assumed to be statistically independent, we have

$$
p_{S_{0}, S_{1}, \ldots, S_{L-1}}\left(s_{0}, s_{1}, \ldots, s_{L-1}\right)=\prod_{l=0}^{L-1} p_{S_{l}}\left(s_{l}\right)
$$

and the average BER for a given $K_{h}$ can be expressed as

$$
\begin{aligned}
P_{b}\left(K_{h}\right)= & \underbrace{\int_{0}^{\infty} \int_{0}^{\infty} \cdots \int_{0}^{\infty}}_{\int^{- \text {fold }}} Q\left(\sqrt{\bar{\gamma} \cdot \sum_{l=0}^{L-1} s_{l}}\right) \\
& \cdot \prod_{l=0}^{L-1} p_{S_{l}}\left(s_{l}\right) d s_{0} d s_{1} \ldots d s_{L-1} \\
= & \underbrace{\int_{0}^{\infty} \int_{0}^{\infty} \cdots \int_{0}^{\infty}} \frac{1}{\pi} \int_{0}^{\pi / 2} \exp \left(-\frac{\bar{\gamma} \sum_{l=0}^{2} s_{l}}{2 \sin ^{2} \theta}\right) d \theta \\
= & \cdot \prod_{l=0}^{L-1} p_{S_{l}}\left(s_{l}\right) d s_{0} d s_{1} \cdots d s_{L-1} \prod_{l=0}^{\pi / 2} I_{l}^{L-1}\left(\bar{\gamma}_{l}, \theta\right) d \theta
\end{aligned}
$$

where

$$
I_{l}\left(\bar{\gamma}_{l}, \theta\right)=\int_{0}^{\infty} \exp \left(-\frac{\bar{\gamma} s_{l}}{2 \sin ^{2} \theta}\right) p_{S_{l}}\left(s_{l}\right) d s_{l} .
$$

Upon substituting (22) into (24), it can be shown that

$$
I_{l}\left(\bar{\gamma}_{l}, \theta\right)=\left(\frac{2 m \sin ^{2} \theta}{\bar{\gamma}_{l}+2 m \sin ^{2} \theta}\right)^{m}
$$

where $\bar{\gamma}_{l}=\bar{\gamma} \lambda_{l}=\bar{\gamma} e^{-\eta l}$. Finally, the average BER can be obtained by substituting (25) into (23), yielding

$$
P_{b}\left(K_{h}\right)=\frac{1}{\pi} \int_{0}^{\pi / 2} \prod_{l=0}^{L-1}\left(\frac{2 m \sin ^{2} \theta}{\bar{\gamma}_{l}+2 m \sin ^{2} \theta}\right)^{m} d \theta .
$$

If we assume that $\eta=0$, then each path has the same MIP distribution and hence $\bar{\gamma}_{0}=\bar{\gamma}_{1}=\ldots=\bar{\gamma}_{L-1}=\bar{\gamma}$ in (26). Therefore, (26) can be expressed as

$$
P_{b}\left(K_{h}\right)=\frac{1}{\pi} \int_{0}^{\pi / 2}\left(\frac{2 m \sin ^{2} \theta}{\bar{\gamma}+2 m \sin ^{2} \theta}\right)^{m L} d \theta .
$$

According to the analysis of Alouini and Goldsmith in [14, Appendix 2], (27) can be expressed in a closed-form as

$$
\begin{aligned}
P_{b}\left(K_{h}\right)= & \sqrt{\frac{\bar{\gamma}}{\bar{\gamma}+2 m}} \frac{(1+\bar{\gamma} / 2 m)^{-m L} \Gamma(m L+1 / 2)}{2 \sqrt{\pi} \Gamma(m L+1)} \\
& \times{ }_{2} F_{1}\left(1, m L+\frac{1}{2} ; m L+1 ; \frac{2 m}{2 m+\bar{\gamma}}\right)
\end{aligned}
$$

where ${ }_{2} F_{1}(a, b ; c ; z)$ is the hypergeometric function defined in $[15]$ as

$$
\begin{aligned}
{ }_{2} F_{1}(a, b ; c ; z) & =\sum_{k=0}^{\infty} \frac{(a)_{k}(b)_{k} z^{k}}{(c)_{k} k !} \text { and } \\
(a)_{k} & =a(a+1) \cdots(a+k-1), \quad(a)_{0}=1 .
\end{aligned}
$$

Consequently, the average BER of the proposed SFH/MC DS-CDMA system using random FH can be computed by substituting (16) [or (17)] and (26) [or (28)] into (15).

\section{B. Uniform FH Scheme}

For the SFH/MC DS-CDMA system using uniform FH, we assume that the FH patterns of all users are determined jointly under the control of the base station (BS), in order that each subcarrier is activated by a similar number of users. It can be shown that for the down-link (base-to-mobile) uniform FH can be readily implemented, since the BS has the knowledge of the FH patterns of all users. However, for implementing up-link (mobile-to-base) transmissions, the FH patterns must be signaled by the BS to each mobile station (MS), in order to be able to implement uniform FH. The advantage of the SFH/MC DS-CDMA system using uniform FH is that the number of users activating each subcarrier is nearly constant, which is lower than the number of active users, $K$. We assume that each user can support $M, 1 \leq M \leq Q$, number of different-rate services by employing $U_{1}, U_{2}, \ldots, U_{M}$ subcarriers. As we analyzed previously, the average number of subcarriers activated by each user is $(1 / M) \sum_{i=1}^{M} U_{i}$, while the average number of users activating each subcarrier can be expressed as

$$
\bar{K}=\frac{K}{Q M} \sum_{i=1}^{M} U_{i}
$$

According to (29), we have $K / Q \leq \bar{K} \leq K$, where $K / Q$ users activate each subcarrier, when each user only activates one subcarrier for transmission at the lowest information rate, i.e., when $M=1, U_{1}=1$. By contrast, $K$ users reside on each subcarrier, when each user activates all the $Q$ number of subcarriers for transmitting at the highest information rate, resulting in $M=1$, $U_{1}=Q$. Note that under the assumption of $M=1, U_{1}=Q$, the SFH/MC DS-CDMA system is actually reduced to a conventional MC DS-CDMA system. As a further advantage, it can be shown that upon using a multiuser detector, the complexity of the SFH/MC DS-CDMA system is significantly lower, than that of the corresponding conventional SCDS-CDMA system or that of the conventional MC DS-CDMA system, provided that each user activates a fraction of the subcarriers for the transmission. Again, the disadvantage of the SFH/MC DS-CDMA system using uniform $\mathrm{FH}$ is however that the side information in the context of FH patterns has to be explicitly signaled to the mobiles, which, to some extent, decreases the system's capacity. According to the above analysis and the analysis in Section III-A, it can be shown that random FH can be employed for the up-link, in order to benefit from both the FH and DS spreading schemes, while uniform FH can be employed for the down-link, in order to achieve low complexity for the multiuser detectors of the MS.

The BER of the proposed SFH/MC DS-CDMA system using uniform FH can be approximated by (26) (or (28), if $\eta=0$ ) 
with the corresponding number of interfering users, $K_{h}$, in (20) replaced by $\bar{K}-1$. Alternatively, (29) can be written as

$$
\bar{K}=K_{I}+P_{h}\left(K_{I}+1\right)
$$

where $K_{I}$ represents the integer part of $\bar{K}$, while $P_{h}\left(K_{I}+1\right)$ is the decimal part of $\bar{K}$ representing the probability that $K_{I}+1$ number of users activate a given subcarrier. Hence, the probability that $K_{I}$ number of users activate a given subcarrier can be expressed as $1-P_{h}\left(K_{I}+1\right)$. Consequently, the average BER of the SFH/MC DS-CDMA system using uniform FH can be expressed as

$$
P_{b}=\left[1-P_{h}\left(K_{I}+1\right)\right] P_{b}\left(K_{I}-1\right)+P_{h}\left(K_{I}+1\right) P_{b}\left(K_{I}\right)
$$

where $P_{b}()$ is given by (26), or (28) if $\eta=0$.

In this section, we have derived the expressions for estimating the BER of the SFH/MC DS-CDMA system considered. Assuming BPSK modulation, the BER of both conventional SC DS-CDMA systems and that of the conventional MC DS-CDMA systems can be estimated by (26) with $K_{h}$ replaced by $K$, the number of the active users. However, in order to make a fair comparison between the SFH/MC DS-CDMA system and the conventional SC DS-CDMA as well as MC DS-CDMA systems, the corresponding parameters must be appropriately adjusted. Our comparison is carried out under the assumption that all schemes occupy the same system bandwidth and use the same transmitted energy per bit. Let $N_{1}=T_{b} / T_{c 1}$ be the spreading gain of a corresponding SC DS-CDMA system, where $T_{c 1}$ represents the chip-duration of the corresponding SC DS-CDMA signal. Moreover, we assume that each subcarrier signal occupies an equal bandwidth and that the total system bandwidth is evenly divided amongst the $Q$ number of subcarriers. Hence, we have $2 / T_{c 1}=Q \times 2 / T_{c}$ or $T_{c}=Q T_{c 1}$. The processing gain of the SFH/MC-DS-CDMA scheme is given by $N_{p}=T / T_{c}=U_{k} T_{b} / Q T_{c 1}=U_{k} N_{1} / Q$, since $T=U_{k} T_{b}$. The corresponding processing gain of a conventional MC DS-CDMA system is given by $N_{p}=T / T_{c}$ $=Q T_{b} / Q T_{c 1}=N_{1}$, since $T=Q T_{b}$. Based on the above assumptions, it can be inferred that the SFH/MC DS-CDMA scheme, the conventional SC DS-CDMA scheme as well as the conventional MC DS-CDMA scheme have the same information rate of $1 / T_{b}$ and the same system bandwidth of $2 / T_{c 1}$, which allows their direct comparison in the next section. Note that since $T_{c}=Q T_{c 1}$, hence, for a given frequency-selective fading channel, the number of resolvable paths, $L_{1}$ associated with the conventional SC DS-CDMA system is at least a factor of $\mathrm{Q}$ higher, than that of the corresponding MC DS-CDMA signal, yielding $L_{1} \approx Q L_{p}$. Bearing these relations in mind, let us now compare the performance of the above systems.

\section{NUMERICAL RESULTS}

In this section, the average BER performance of the proposed system is evaluated as a function of the average received SNR per bit, which is obtained by computing

$$
\bar{\gamma}_{b}=\frac{\left(1-e^{-L_{p} \eta}\right)}{1-e^{-\eta}}\left(\frac{\Omega_{0} E_{b}}{N_{0}}\right)=q\left(L_{p}, \eta\right)\left(\frac{\Omega_{0} E_{b}}{N_{0}}\right)
$$

or as a function of the number of active users for the proposed system described above. All results were computed from

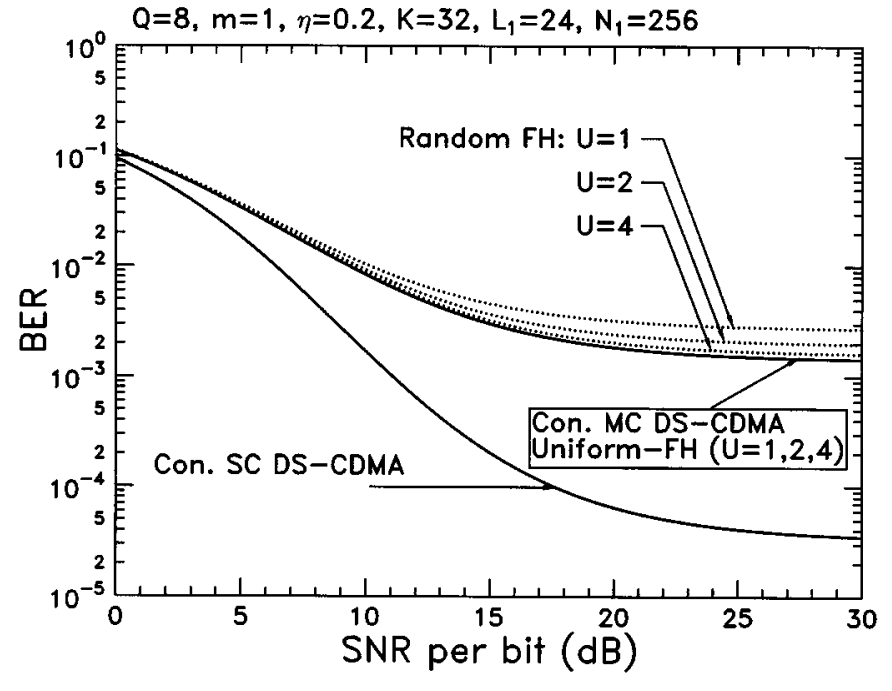

Fig. 3. BER versus SNR per bit performance comparison among the SFH/MC DS-CDMA, conventional SC DS-CDMA and conventional MC DS-CDMA systems over multipath Rayleigh fading channels $(m=1)$.

(15)-(17) and (26). The variables related to our computations are listed below:

$N_{1} \quad$ spreading gain of the corresponding SC DS-CDMA signals;

$L_{1} \quad$ number of resolvable paths of the corresponding SC DS-CDMA signals;

$N \quad$ number of chips per bit before S-P conversion for the SFH/MC DS-CDMA signal;

$Q \quad$ number of subcarriers;

$U \quad$ weight of constant-weight codes;

$C(Q, U)$ constant-weight code with length of $Q$ and weight of $U$;

$L_{p} \quad$ number of resolvable paths;

$L \quad$ order of diversity used;

$K \quad$ number of active users;

$m$ fading parameter of the Nakagami fading channels; $\eta \quad$ MIP decay factor.

In Figs. 3 and 4, we show the comparison of the BER performance of the SFH/MC DS-CDMA systems using both random $\mathrm{FH}$ and uniform $\mathrm{FH}$ with that of the conventional SC DS-CDMA system as well as that of the conventional MC DS-CDMA system. The curves in both figures were plotted against the average SNR per bit under the assumption that the number of resolvable paths and the spreading gain of the corresponding SC DS-CDMA system was $L_{1}=24$ and $N_{1}=256$, respectively. We assumed that the number of subcarriers in both the SFH/MC DS-CDMA system and in the conventional MC DS-CDMA system was $Q=8$. Hence, according to the relationship of $L_{p}=L_{1} / Q$, the number of resolvable paths associated with each subcarrier signal was about $L_{p}=3$. In Fig. 3, we used $m=1$ corresponding to a multipath Rayleigh fading channel model, and $\eta=0.2$ corresponding to a relatively slowly decaying MIP and a severely dispersive fading channel. However, in Fig. 4, we assumed $m=3$, which corresponds to a Ricean multipath fading channel model with a Ricean factor of $\mathcal{K} \approx 4.45$, and $\eta=3$, which corresponds to a relatively low-dispersion fading channel. The results of Fig. 3 show that 


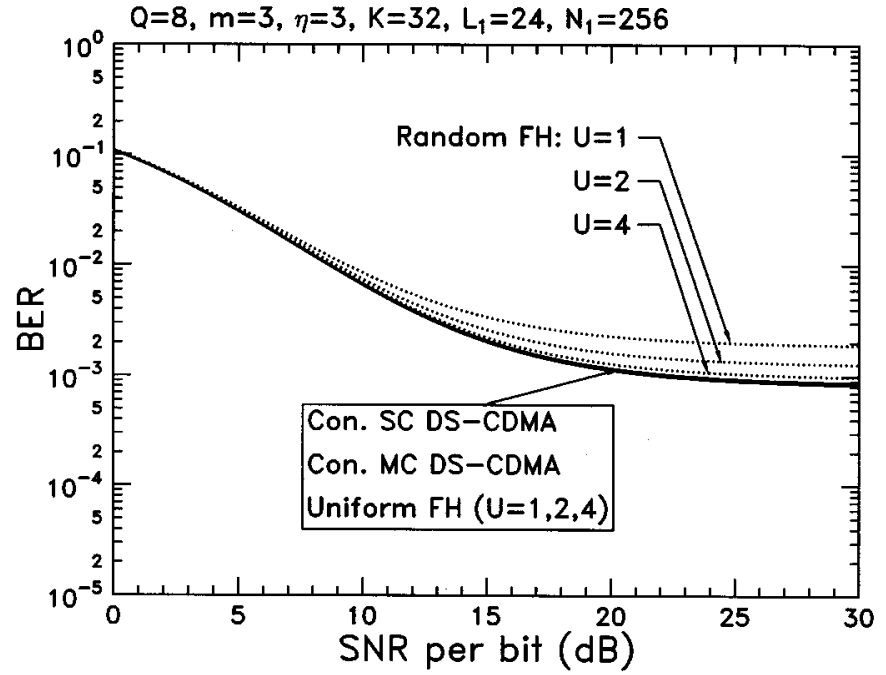

Fig. 4. BER versus SNR per bit performance comparison among the SFH/MC DS-CDMA, conventional SC DS-CDMA and conventional MC DS-CDMA systems over multipath Ricean fading channels, where $m=3$ corresponds to the Ricean factor of $\mathcal{K} \approx 4.45$.

under the assumption of multipath Rayleigh fading exhibiting a severe dispersion, the conventional SC DS-CDMA system achieves the best BER performance, since the conventional SC DS-CDMA system has a significantly higher diversity order of $L_{1}=24$, than the SFH/MC DS-CDMA and the conventional MC DS-CDMA systems, which have a diversity order of $L_{p}=3$. The conventional MC DS-CDMA system has the same BER performance as that of the SFH/MC DS-CDMA system using the uniform $\mathrm{FH}$ scheme, and their BER performance is better than that of the SFH/MC DS-CDMA system using the random FH scheme. For a given SNR per bit value, the BER performance of the SFH/MC DS-CDMA system using the uniform FH scheme remains a constant, irrespective of the number of subcarriers activated. However, for the SFH/MC DS-CDMA system using the random FH scheme, the BER slightly decreases upon increasing the number of active subcarriers. The results of Fig. 4 indicate that, if the communication environment becomes better and a low-dispersion fading channel is assumed, the conventional SC DS-CDMA, the conventional MC DS-CDMA, and the SFH/MC DS-CDMA using the uniform FH scheme achieve almost the same BER. However, for the SFH/MC DS-CDMA system using random $\mathrm{FH}$, the BER decreases upon increasing the number of active subcarriers, as it was observed also in Fig. 3.

In order to show the achievable improvements due to diversity, Figs. 5 and 6 depict the BER of the SFH/MC DS-CDMA system using random $\mathrm{FH}$ as a function of the average received SNR per bit. The individual curves in each figure are parameterized by the diversity order of $L=1,2, \ldots, 6$. Fig. 5 represents the multipath Rayleigh fading environment $(m=1)$, while Fig. 6 characterizes the Ricean multipath fading environment $(m=3)$, where $m=3$ corresponds to a Ricean factor of $\mathcal{K} \approx 4.45$. The other parameters associated with our investigations were shown at the top of the figures. From the results, it is seen that the faster the MIP decays, the faster the incremental diversity improvement decreases. Nonetheless, the system's BER

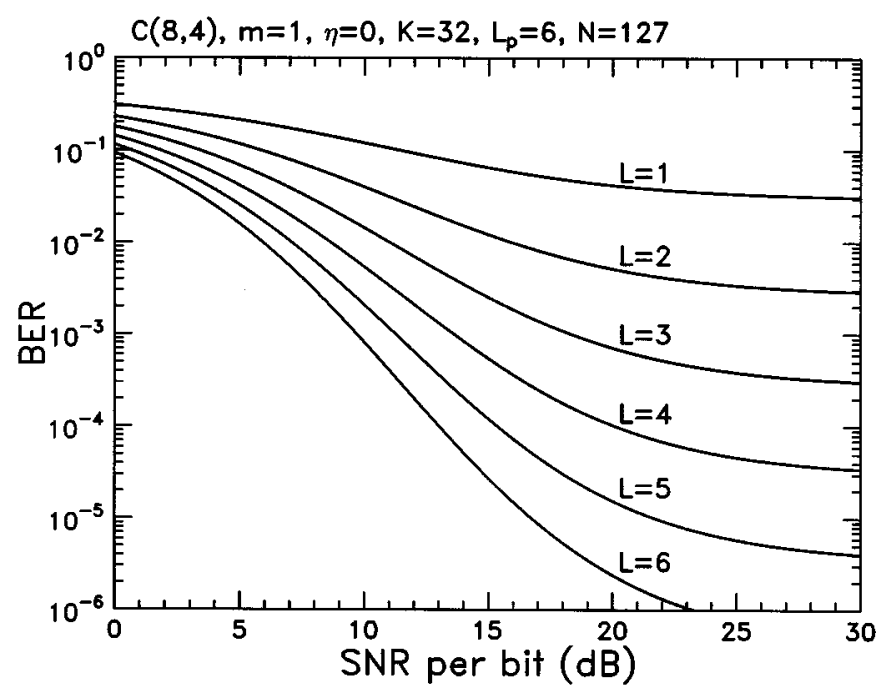

Fig. 5. BER versus SNR per bit performance for the constant-weight code based SFH/MC DS-CDMA system over multipath Rayleigh fading channels ( $m=1$ ) upon varying the diversity order $L$ for a MIP decay factor of $\eta=0$. For the receiver using MRC, the optimum diversity order $L$ is its maximum possible value, namely $L=L_{p}$, combining all the resolvable multipath components.

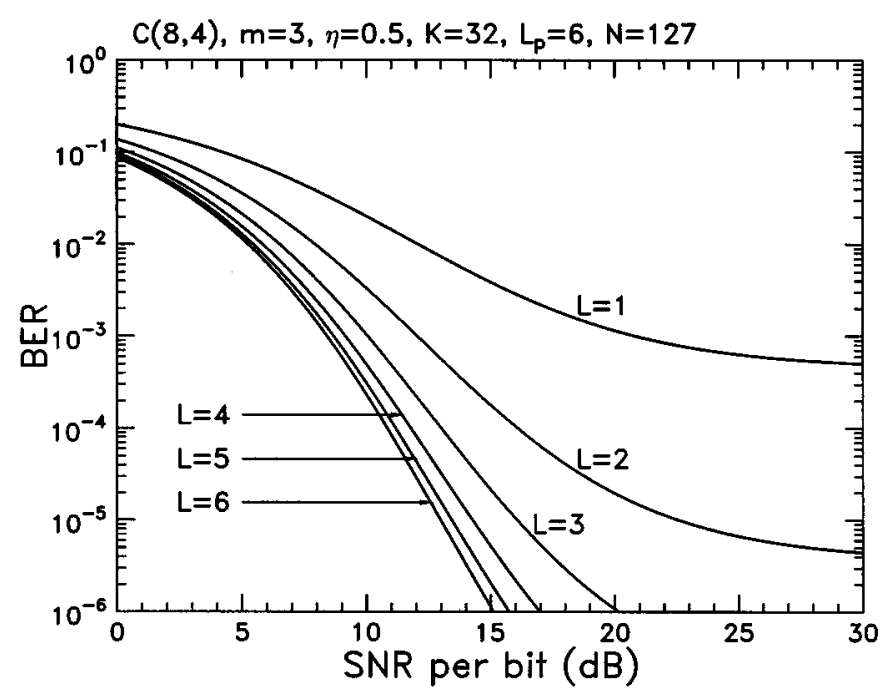

Fig. 6. BER versus SNR per bit performance for the constant-weight code based SFH/MC DS-CDMA system over multipath Ricean fading channels ( $m=3$ ) upon varying the diversity order $L$ for a MIP decay factor of $\eta=0.5$. Best performance is achieved by combining all resolvable multipaths using MRC.

performance is dramatically improved upon increasing the diversity order $L$. For MRC and for the multipath channel having relatively low MIP decay factors, the optimal value of $L$ is seen to be its maximum possible value, namely $L=L_{p}$, which implies combining all the resolvable multipath components, irrespective of the associated complexity.

Fig. 7 shows the BER performance of the SFH/MC DS-CDMA system with respect to the multipath fading parameters $m=0.5,1,2,5,20, \infty$. As noted before, the Nakagami parameter $m$ represents different fading environments, ranging from the worst-case one-sided Gaussian fading to Rayleigh, and finally to the most favorable nonfading AWGN case. As expected, for a given SNR per bit, the BER decreases upon 


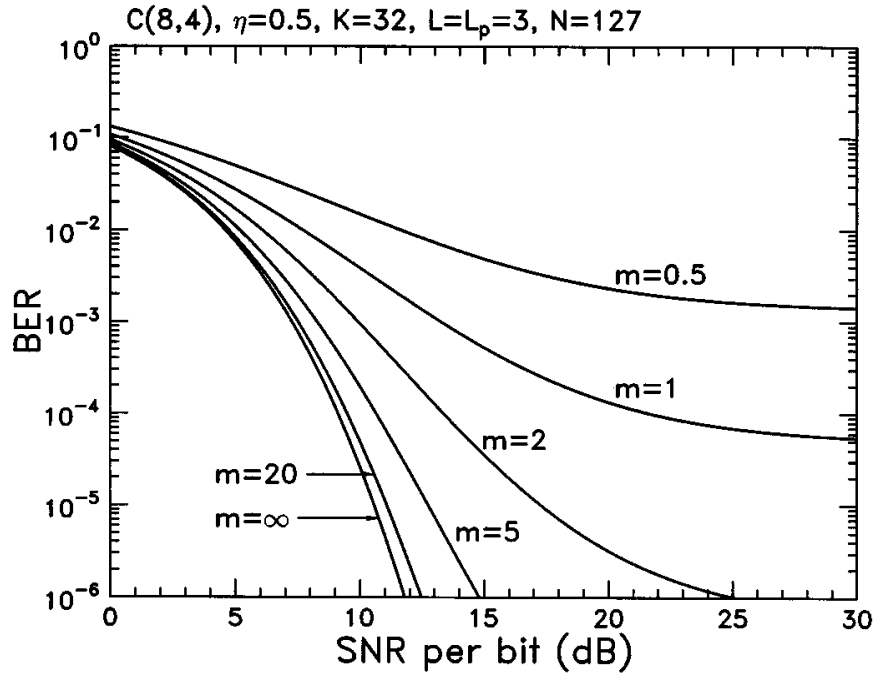

Fig. 7. BER versus SNR per bit performance for the constant-weight code based SFH/MC DS-CDMA system over different fading channels upon varying the Nakagami fading parameter $m$ for a MIP decay factor of $\eta=0.5$. Best performance is achieved over the AWGN channel, namely for $m \rightarrow \infty$.

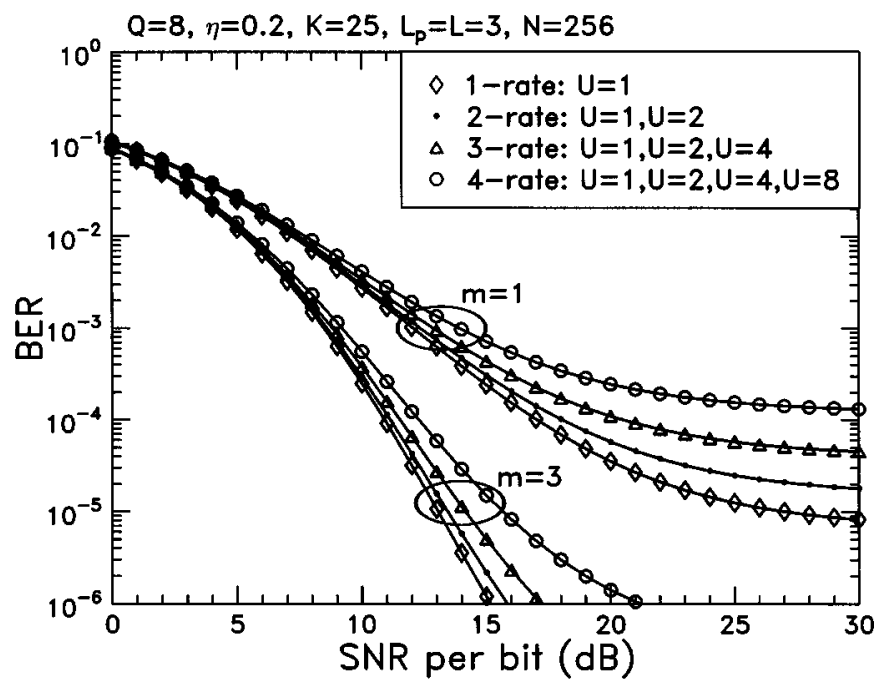

Fig. 8. BER versus SNR per bit performance for the constant-weight code based multirate SFH/MC DS-CDMA system over both multipath Rayleigh fading channels $(m=1)$ and multipath Ricean fading channels $(m=3)$ for $L_{p}=L=3$ upon varying the number of different information rates provided. It is shown that for a given value of $m$ the BER increases, as the number of information rates provided increases.

increasing the value of $m$, which implies that the channel fading becomes less severe. The system performance is critically affected by the parameter $m$, i.e., the Nakagami communication environment encountered.

The BER performance of the constant-weight code-based multirate SFH/MC DS-CDMA systems using random FH schemes is shown in Figs. 8 and 9 in terms of the multipath Nakagami- $m$ fading channels having different fading parameters of $m$ (Fig. 8) and different number of active users (Fig. 9). In Fig. 8, we assumed $m=1$ corresponding to multipath Rayleigh fading and $m=3$ corresponding to multipath Ricean fading. In Figs. 8 and 9, a single-rate (1-rate) is supported by employing a weight- 1 constant-weight code $\mathrm{C}(8,1)$, while a twin-rate (2-rate) system is supported by the constant-weight

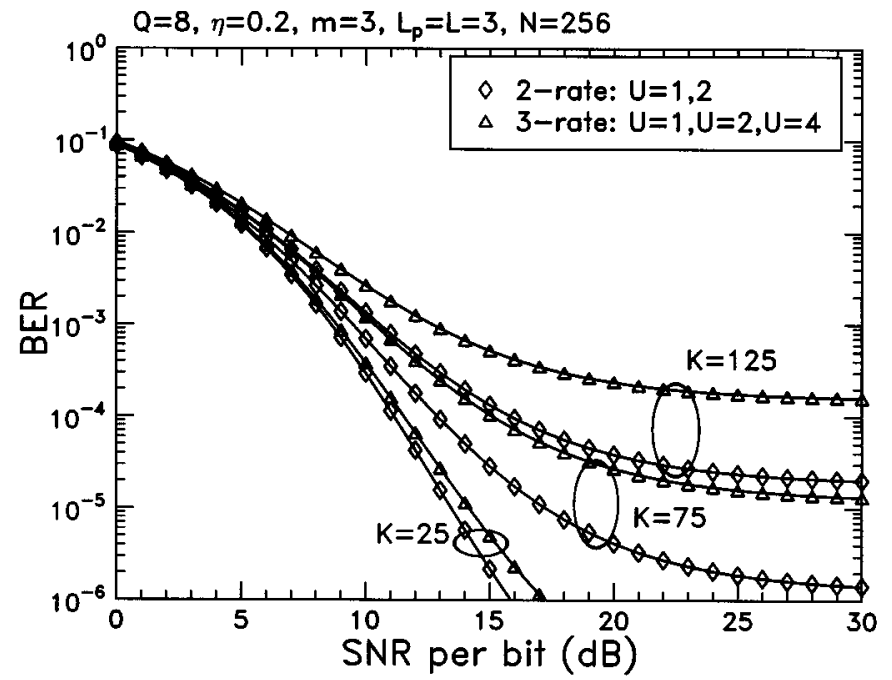

Fig. 9. BER versus SNR per bit performance for the constant-weight code based multirate SFH/MC DS-CDMA system over multipath Ricean fading channels ( $m=3$ ) for $L_{p}=L=3$ upon varying the number of different information rates provided and the number of active users, $K$. It is shown that for a given value of $K$, the BER increases, as the number of information rates provided increases.

codes $\mathrm{C}(8,1)$ and $\mathrm{C}(8,2)$. Similarly, a triple-rate (3-rate) system is created by $C(8,1), C(8,2)$ and $C(8,4)$, and a quadruple-rate (4-rate) system is generated by the codes $\mathrm{C}(8,1), \mathrm{C}(8,2), \mathrm{C}(8,3)$, and $\mathrm{C}(8,4)$. Let $R_{b}$ be the information rate provided by a single subcarrier. Since the symbol duration of the multirate SFH/MC DS-CDMA system was assumed to be a constant, i.e., $T=T_{b}$, the information rates supported by $\mathrm{C}(8,1), \mathrm{C}(8,2), \mathrm{C}(8,4)$, and $\mathrm{C}(8,8)$, hence are $R_{b}, 2 R_{b}, 4 R_{b}$, and $8 R_{b}$, respectively. From the results of Figs. 8 and 9, we observe that for a given fading parameter $m$ in Fig. 8 or for a given number of active users, $K$ in Fig. 9, the system performance degrades, as the number of information rates supported increases. Taking $m=3$ in Fig. 8 as an example, the transmitted energy per bit must be increased by about $0.8 \mathrm{~dB}$, in order that the system can support the information rates of $R_{b}$ and $2 R_{b}$ (dot), instead of the information rate of $R_{b}$ (diamond), while maintaining a BER of $10^{-6}$. Similarly, a further $1.1-\mathrm{dB}$ or $4-\mathrm{dB}$ transmitted energy per bit must be invested, in order to upgrade the system from a twin-rate to triple-rate or from triple-rate to quadruple-rate, respectively. Note that since a higher information rate requires a higher number of subcarriers, this results in the decrease of the subcarrier-SNR. Hence, in order to maintain a constant BER, the subcarrier-SNR reduction must be compensated by increasing the transmitted energy per bit. However, due to the MAI-induced error floor, the associated SNR loss sometimes cannot be compensated by simply increasing the transmitted power, as seen in Fig. 8 for $m=1$ in the BER range of $10^{-5}$ to $10^{-4}$. The results of Fig. 9 also show that the BER performance of SFH/MC DS-CDMA is significantly degraded, when the number of active users increases.

Finally, in Fig. 10, we show that for a receiver combining a given number of resolvable paths, the average BER can be minimized by adjusting the number of subcarriers. In Fig. 10, SFH/MC DS-CDMA systems having a constant system bandwidth associated with $N Q=2^{10}$, but using various combina- 


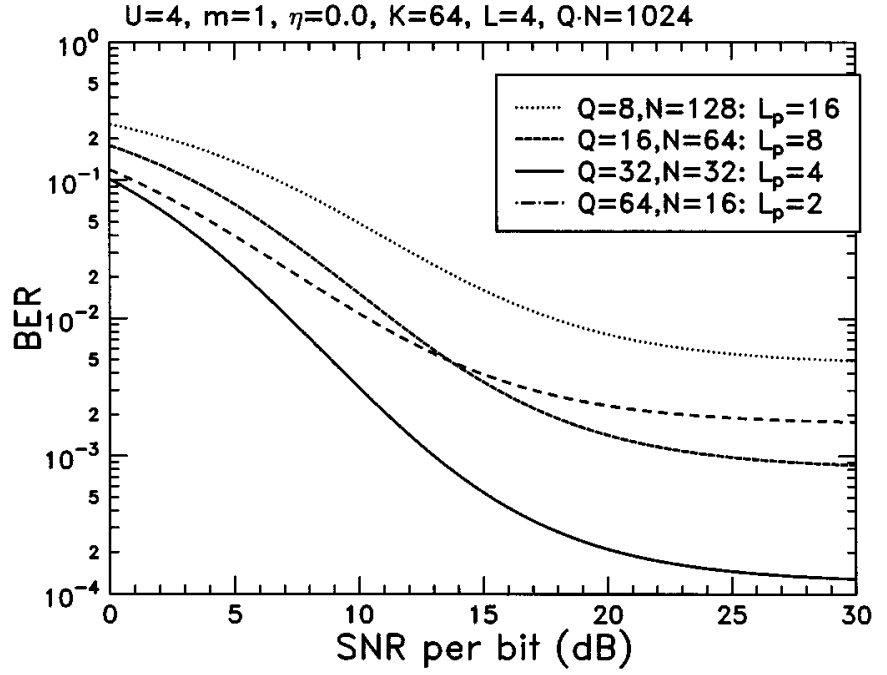

Fig. 10. BER versus SNR per bit performance for the constant-weight code-based SFH/MC DS-CDMA system upon varying the value of $N$ and $Q$ for a maximum diversity order of $L=4$ and $L_{p}=2,4,8,16$. Given that the number of paths combined was $L=4$, the system with $Q=32, N=32$, $L=L_{p}=4$ achieved the best performance.

tions of the number of subcarriers $Q$ and bit-duration to chip-duration ratio of $N$ were considered. In this system, increasing the number of subcarriers implies decreasing the 'hit' probability inflicted by the interfering users and simultaneously, decreasing the direct-sequence spread bandwidth of each subcarrier. We assumed that a receiver had a maximum diversity order of $L=4$, although a higher number of resolvable paths, $L_{p}$, was available at the receiver. From the results, we infer that the curve associated with $Q=32, N=32$, or $L=L_{p}=4$ achieves the best BER performance. Therefore, for a SFH/MC DS-CDMA system with a constant system bandwidth, the system can be optimized. Specifically, if the receiver is capable of combining a given number of multipaths, namely $L$, then according to the average delay spread of the channel, the DS spread bandwidth of each subband can be adjusted, so that the resulting number of resolvable paths, $L_{p}$, is as close to $L$ as possible. Then, the receiver can efficiently utilize the energy dispersed over the multipath components. The required number of subbands consequently can be obtained by dividing the system bandwidth by the DS spread bandwidth necessitated.

\section{CONCLUSION}

The proposed SFH/MC DS-CDMA system can amalgamate efficiently the techniques of slow FH, OFDM, and DS-CDMA. Nonlinear constant-weight codes have been introduced, in order to control the associated FH patterns and to efficiently share the system's frequency resources by each user. Furthermore, constant-weight codes with different weights can be employed to activate a number of subcarriers, in order to support multirate services. The performance of the proposed system using a coherent RAKE receiver with MRC has been evaluated and compared with that of corresponding SC DS-CDMA systems and that of corresponding MC DS-CDMA systems, when these systems operated in a multipath Nakagami fading environment, and when the Gaussian approximation was invoked for the multi- path and MAI. From the results, we concluded that the proposed SFH/MC DS-CDMA is an attractive wireless multiple access candidate, which is capable of interworking with the existing $2 \mathrm{G}$ and $3 \mathrm{G}$ CDMA systems, while providing an evolutionary path for future unlicensed and BRAN networks without rigid and unnecessary spectrum fragmentation.

\section{ACKNOWLEDGMENT}

The authors wish to thank the anonymous reviewers for their constructive suggestions and comments.

\section{REFERENCES}

[1] E. Dahlman, B. Gudmundson, M. Nilsson, and J. Skold, "UMTS and IMT-2000 based on wideband CDMA," IEEE Commun. Mag., vol. 36, pp. 70-80, Sept. 1998.

[2] L.-L. Yang and L. Hanzo, "Blind soft-detection assisted frequency-hopping multicarrier DS-CDMA," in Proc. IEEE GLOBECOM, Rio de Janeiro, Brazil, Dec. 5-9,, 1999, pp. 842-846.

[3] — - "Blind joint soft-detection assisted slow frequency-hopping multicarrier DS-CDMA," IEEE Trans. Commun., vol. 48, pp. 1520-1529, Sept. 2000

[4] E. A. Sourour and M. Nakagawa, "Performance of orthogonal multicarrier CDMA in a multipath fading channel," IEEE Trans. Commun., vol. 44, pp. 356-367, Mar. 1996.

[5] Y. Sanada and M. Nakagawa, "A multiuser interference cancellation technique utilizing convolutional codes and orthogonal multicarrier modulation for wireless indoor communications," IEEE J. Select. Areas Commun., vol. 14, pp. 1500-1509, Oct. 1996.

[6] Q. Chen, E. S. Sousa, and S. Pasupathy, "Multicarrier CDMA with adaptive frequency hopping for mobile radio systems," IEEE J. Select. Areas Commun., vol. 14, pp. 1852-1858, Dec. 1996.

[7] S. Kondo and L. B. Milstein, "Performance of multicarrier DS CDMA systems," IEEE Trans. Commun., vol. 44, pp. 238-246, Feb. 1996

[8] L. Vandendorpe, "Multitone spread spectrum multiple access communications system in a multipath Rician fading channel," IEEE Trans. Veh. Technol., vol. 44, pp. 327-337, May 1995.

[9] D. N. Knisely, S. Kumar, S. Laha, and S. Nanda, "Evolution of wireless data services: IS-95 to CDMA 2000," IEEE Commun. Mag., vol. 36, pp. 140-149, Oct. 1998.

[10] T. Vlachos and E. Geraniotis, "Performance study of hybrid spread-spectrum random-access communications," IEEE Trans. Commun., vol. 39, pp. 975-985, June 1991.

[11] J. Wang and M. Moeneclaey, "Hybrid DS/SFH-SSMA with predetection diversity and coding over indoor radio multipath Rician-fading channels," IEEE Trans. Commun., vol. 40, pp. 1654-1662, Oct. 1992.

[12] N. Nakagami, "The $m$-distribution, a general formula for intensity distribution of rapid fading," in Statistical Methods in Radio Wave Propagation, W. G. Hoffman, Ed. Oxford, U.K.: Pergamon, 1960.

[13] M. K. Simon and M. Alouini, "A unified approach to the probability of error for noncoherent and differentially coherent modulations over generalized fading channels," IEEE Trans. Commun., vol. 45, pp. 1625-1638, Dec. 1998.

[14] M.-S. Alouini and A. J. Goldsmith, "A unified approach for calculating error rates of linearly modulated signals over generalized fading channels," IEEE Trans. Commun., vol. 47, pp. 1324-1334, Sept. 1999.

[15] T. Eng and L. B. Milstein, "Coherent DS-CDMA performance in Nakagami multipath fading," IEEE Trans. Commun., vol. 43, pp. 1134-1143, Feb. 1995.

[16] L. L. Yang and C. S. Li, "DS-CDMA performance of random orthogonal codes over Nakagami multipath fading channels," in Proc. 4th IEEE ISSSTA, Mainz, Germany, Sept. 22-25, 1996, pp. 68-72.

[17] G. P. Efthymoglou, V. A. Aalo, and H. Helmken, "Performance analysis of coherent DS-CDMA systems in a Nakagami fading channel with arbitrary parameters," IEEE Trans. Veh. Technol., vol. 46, pp. 289-296, May 1997.

[18] _ - "Performance analysis of noncoherent DS-CDMA systems in a Nakagami fading channel with arbitrary parameters," in IEE Proc. Commun., vol. 144, June 1997, pp. 166-172.

[19] F. Patenaude, J. H. Lodge, and J. Y. Chouinard, "Noncoherent diversity reception over Nakagami-fading channels," IEEE Trans. Commun., vol. 46, pp. 985-991, Aug. 1998. 
[20] E. K. Al-Hussaini and A. M. Al-Bassiouni, "Performance of MRC diversity systems for the detection of signals with Nakagami fading," IEEE Trans. Commun., vol. 33, pp. 1315-1319, Dec. 1985.

[21] J. G. Proakis, Digital Communications, 3rd ed. New York: McGrawHill, 1995.

[22] S. Verdu, Multiuser Detection. Cambridge, U.K.: Cambridge Univ. Press, 1998

[23] R. K. Morrow and J. S. Lehnert, "Bit-to-bit error dependence in slotted DS/SSMA packet systems with random signature sequences," IEEE Trans. Commun., vol. 37, pp. 1052-1061, Oct. 1989.

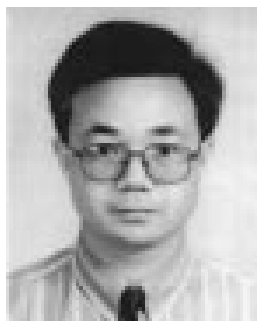

Lie-Liang Yang (M'98) received the B.Eng. degree in communication engineering from Shanghai TieDao University, Shanghai, China, in 1988, and the M.S and Ph.D. degrees in communications and electronics from Northern Jiaotong University, Beijing, China, in 1991 and 1997, respectively.

From 1991 to 1993, he was a Lecturer with the Department of Electrical Engineering, East-China Jiaotong University, China. From 1993 to 1997, he was with the Modern Communications Research Institute, Northern Jiaotong University, Nanchang, China. From June 1997 to December 1997, he was a Visiting Scientist with the Institute of Radio Engineering and Electronics, Academy of Sciences of the Czech Republic, Praque. Since December 1997, he has been with the Communication Group, Department of Electronics and Computer Science, University of Southampton, U.K., and has been involved in researching various error-correction coding, modulation, and detection techniques, as well as wideband, broadband, and ultra wideband CDMA systems for the advanced wireless mobile communication systems. He has published over 60 papers in journals and conference proceedings.

Dr. Yang was awarded the Royal Society Sino-British Fellowship in 1997.

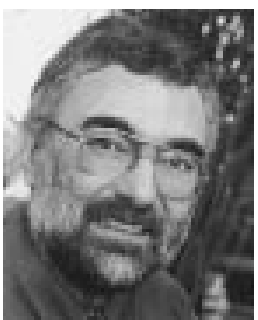

Lajos Hanzo (M'91-SM'92) received a Masters degree in electronics in 1976 and the Ph.D. degree in 1983, both from the Technical University of Budapest, Budapest, Hungary.

During his 24-year career in telecommunications, he has held various research and academic posts in Hungary, Germany, and the U.K. Since 1986, he has been with the Department of Electronics and Computer Science, University of Southampton, U.K., and has been a Consultant with Multiple Access Communications Ltd., Southampton, U.K. Currently, he holds a chair in telecommunications. He coauthored five books on mobile radio communications, published in excess of 300 research papers, organized and chaired conference sessions, presented overview lectures, and was awarded a number of distinctions. Currently, he is managing an academic research team, working on a range of research projects in the field of wireless multimedia communications sponsored both by industry and under the auspices of the Engineering and Physical Sciences Research Council (EPSRC), U.K., the European IST Programme, and the Mobile Virtual Centre of Excellence (VCE), U.K. 